\title{
OSCILLATORY STRAIN SWEEPS OF HYDROGELS FROM METHACRYLATED ALGINATE MACROMONOMERS: ASSESSMENT OF SYNTHESIS AND ACQUISITION VARIABLES
}

\author{
CAMILA SOLAR, DANIEL PALACIO, SUSANA SÁNCHEZ AND BRUNO F. URBANO* \\ Departamento de Polímeros, Facultad de Ciencias Químicas, Universidad de Concepción, Concepción, Chile.
}

\begin{abstract}
The objective of this study is the rheological characterization of photocrosslinked alginate hydrogels synthesized from methacrylated alginate macromonomers. We first optimized the rheological acquisition parameters for this hydrogels, that is, we studied the influence of the geometries diameter, distance between plates (gap), and solvent trap in the rheological response. Our results show important variations of shear storage modulus, G', and yield strain with the three parameters measured. Secondly, with the optimized experimental parameters, rheological tests were performed on hydrogels synthesized under different conditions. Three parameters were studied; UV exposure time (polymerization time), macromonomer concentration and photoinitiator concentration. Our data indicate that the polymerization time and the macromonomer concentration have an important effect on the viscoelastic properties of the hydrogel, significantly modifying the shear storage modulus, G', and the yield strain while the photoinitiator concentration did not influence the viscoelastic properties.
\end{abstract}

Keywords: rheology, alginate, hydrogel, macromonomer.

\section{INTRODUCTION}

Hydrogels are crosslinked polymers able to absorb water without dissolving. The crosslinking can be of chemical origin through covalent bonds or physical by weak interactions (such as dipole-dipole type interactions, hydrogen bonds, etc) ${ }^{1}$. These materials have found applications in various areas such as hygiene products, adsorbents, sensors among others, however, the most promising applications are in the area of biomedicine $e^{2-4}$. This is due to the fact that their capacity to hydrate, exchange mass with the environment and their viscoelastic properties, make hydrogels an attractive alternative to mimic the extracellular matrix 5 .

The hydrogels may be constituted of synthetic polymers or biopolymers, or combinations of these. The former are obtained generally from vinyl monomers (e. g. acrylamide, 2-hydroxyethyl methacrylate, acrylic acid, among others $)^{6-8}$, and in general, require the use of a cross-linking agent that keeps the polymer chains covalently bound to provide dimensional stability in a swollen state. On the other hand, hydrogels obtained from biopolymers such as alginate ${ }^{9}$, gelatin ${ }^{10}$, carrageenan ${ }^{11}$, among others, are more attractive for biomedical applications since, due to their natural origin, they provide greater biocompatibility and biodegradation ${ }^{12}$. However, the control of the structure is more limited than in synthetic polymers because of the complexity of its structure.

Alginate is an unbranched linear polysaccharide obtained from brown marine algae (Laminaria hyperborea, Laminaria digitata, Laminaria japonica, Ascotyllum nodosum, and Macrocystis pyrifera), through an extraction with sodium hydroxide. Structurally, the alginate corresponds to a linear copolymer that has repeating units of $\beta$-D-mannuronic acid $(\mathrm{M})$ and $\alpha$-L-guluronic acid $(\mathrm{G})$ linked through 1,4-glycosidic bonds and which is distributed in consecutive blocks of $\mathrm{G}$ or $\mathrm{M}$ and blocks with alternating residues ${ }^{9}$. Alginate is recognized due to its structural characteristics that allow obtaining hydrogels, in addition to its biocompatibility, biodegradability and low cost. One of the ways to obtain gels is through the formation of ionic crosslinking with divalent ions such as $\mathrm{Ca}^{2+}$. In this case, the $\mathrm{G}$ blocks possess a high degree of coordination with the divalent cations, which added to blocks of $\mathrm{G}$ of an adjacent chain form an eggbox structure that acts as a crosslinking site giving rise to the gel. Also, to form covalently crosslinked structures it is possible to make modifications in the structure of the alginate, functionalizing with polymerizable vinyl groups and allowing crosslinking. Among the different forms of obtaining hydrogels by chemical crosslinking, one of them is through photopolymerization, where this reaction is carried out by placing a polymer precursor (macromonomer) in light in the presence of a photoinitiator where the polymerization occurs too quickly allowing a reticulation in situ $^{13}$. This method uses ultraviolet (UV) light to generate the free radicals that allow forming covalent bonds between the functional methacrylate groups along the entire polymer chain.

The objective of this research is to study the variable effect of the synthesis of hydrogels in the rheological properties. The hydrogel will consist of sodium alginate and will be obtained from alginate macromonomers synthesized through a methacrylation reaction. Similarly, analysis parameters such as diameter of geometries, space between geometry and peltier (gap), and the use of solvent trap to this type of materials will also be studied.

\section{MATERIALS AND METHODS}

\subsection{Synthesis of the alginate macromonomers and hydrogels}

The macromonomer of methacrylated alginate was obtained through the esterification of hydroxyl groups as proposed by Zhao et al. ${ }^{17}$. To this, $2.0 \mathrm{~g}$ of sodium alginate (Sigma-Aldrich) was solubilized in $100 \mathrm{~mL}$ of deionized water under constant agitation and room temperature. Subsequently the $\mathrm{pH}$ was adjusted to 8.0 using an aqueous solution of $\mathrm{NaOH}$ (Sigma-Aldrich, 99\%) at a temperature of $4{ }^{\circ} \mathrm{C}$ for $20 \mathrm{~min}$, then methacrylic anhydride $41.1 \mathrm{~mL}(0.27 \mathrm{~mol})$ (Sigma-Aldrich, 94\% ) was slowly added until obtaining a homogeneous solution which was maintained for $24 \mathrm{~h}$ at $4{ }^{\circ} \mathrm{C}$ and at a constant $\mathrm{pH}$ of 8.0. Finally, the methacrylated alginate solution (AlgMA) was dialyzed in distilled water for 96 $\mathrm{h}$ using cellulose bags with a molecular weight cut-off of 12,000 to $14,000 \mathrm{~g} /$ mol and subsequently lyophilized.

The hydrogels were obtained through photopolymerization of AlgMa macromonomers using 2-hydroxy-4'-(2-hydroxyethoxy)-2methylpropiophenone as initiator (Irgacure 2959, Sigma-Aldrich, 98\%) in a UVcrosslinker device (UVP model CL1000) at a wavelength of $365 \mathrm{~nm}$. To that end, a certain amount of AlgMA was solubilized in deionized water, the photoinitiator was added and the solution was degassed under a nitrogen atmosphere. A volume of the solution was placed in Teflon molds with a diameter of $8.0 \mathrm{~mm}$ and a depth of $2.0 \mathrm{~mm}$ and covered with a silinized slide to polymerize. The synthesis variables studied were: AlgMA concentration, Irgacure concentration and polymerization time (see table 1)

Table 1. Variables of AlgMA hydrogel.

\begin{tabular}{|c|c|c|}
\hline $\begin{array}{c}\text { AlgMA } \\
(\boldsymbol{\%} \mathbf{m} / \mathbf{v})\end{array}$ & $\begin{array}{c}\text { Time } \\
(\mathbf{m i n})\end{array}$ & $\begin{array}{c}\text { Irgacure 2959 } \\
(\mathbf{\%} \mathbf{m} / \mathbf{v})\end{array}$ \\
\hline 1.0 & 5.0 & 0.05 \\
\hline 2.0 & 10.0 & 0.1 \\
\hline 3.0 & 20.0 & - \\
\hline
\end{tabular}

\section{Physicochemical characterization}

Infrared spectroscopy with Fourier transform (FTIR): the spectra were obtained using a Nicolet spectrophotometer Magna 550 model. The samples were analyzed in the mid-infrared $400-4000 \mathrm{~cm}-1$ in a solid sample solution with $\mathrm{KBr}$.

Nuclear magnetic resonance $\left({ }^{1} \mathrm{H}-\mathrm{NMR}\right)$ : The analysis was carried out by dissolving alginate and macromonomer in $\mathrm{D}_{2} \mathrm{O}$ at a concentration of $10 \mathrm{mg} / \mathrm{mL}$, and the spectra were acquired using a Bruker Avance $400 \mathrm{MHz}$ spectrometer. 
Swelling capacity: the water absorption capacity of AlgMA hydrogels was evaluated by immersing hydrogel discs $(8 \mathrm{~mm} \times 2 \mathrm{~mm})$ in distilled water at room temperature and determining the mass of the swollen discs at different intervals of time. The capacity was determined through the mass difference of the swollen and dehydrated gels by the following expression:

$$
S_{w}=\frac{w_{s}-w_{d}}{w_{d}}
$$

Where $\mathrm{w}_{\mathrm{s}}$ is the dry hydrogel mass and $\mathrm{w}_{\mathrm{s}}$ is the mass of the swollen hydrogel.

Rheology: The viscoelastic properties of the hydrogels were analyzed in a Hybrid rheometer TA Instrument model DHR-3 equipped with Peltier system for temperature control. We performed strain sweep tests on hydrogel discs after photopolymerization using an $8 \mathrm{~mm}$ diameter geometry in a strain range of 0.01 to $100 \%$ at $1 \mathrm{~Hz}$ and $25^{\circ} \mathrm{C}$. Parameters such as the storage module $\left(\mathrm{G}^{\prime}\right)$ and loss module $\left(G^{\prime \prime}\right)$ were determined to assess the effect of the synthesis variables.
With hydrogel that presented the best results, it was used to study the effect of the analysis variables and determine their effect on the rheological response. To do this, several variables were studied, such as geometry diameter (8.0, 20.0, 25, and $40.0 \mathrm{~mm})$, gap $(1.5$ and $2.0 \mathrm{~mm})$, roughness of the geometry and the solvent trap.

\section{RESULTS AND DISCUSSION}

\subsection{AlgMA macromonomer characterization.}

The macromonomer (Alg MA) was obtained by the esterification of alginate (Alg) with methacrylic anhydride ${ }^{14}$. In the methacrylation reaction of the alginate (Fig 1) there is a nucleophilic attack of the hydroxyl groups (on the sp2 carbon of the carbonyl) obtaining methacrylic acid as a by-product. In this manner, the alginate chains will contain methacrylate groups used in the polymerization process via free radicals. The alginate reaction occurs preferentially in the $\mathrm{C}-2$ hydroxyl group of a mannuronic acid residue, however, taking into account its similar reactivity, the C-3 hydroxyl group of mannuronic acid is also prone to methacrylation, in addition to the residues hydroxyl present in the guluronic acid units (see Figure 1$)^{15}$.
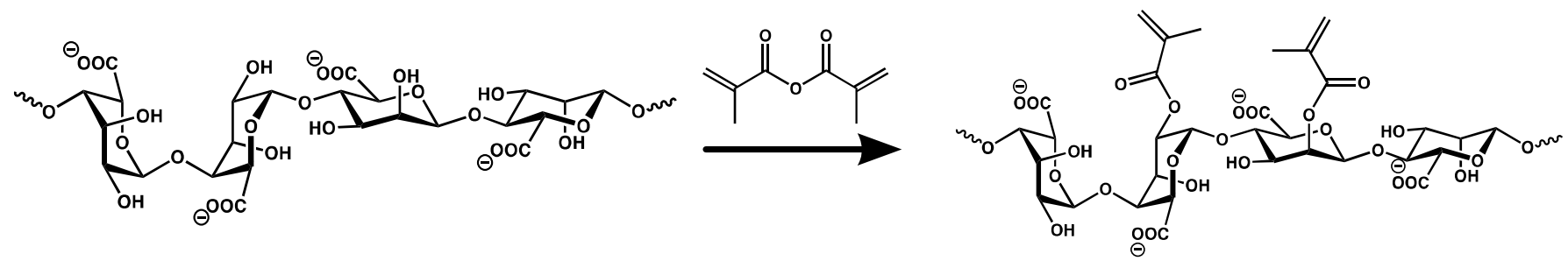

Figure 1. Scheme of methacrylated macromonomer synthesis.

The resulting macromonomer (AlgMA) was characterized by Fourier Transformed Infrared spectroscopy (FTIR), and ${ }^{1} \mathrm{H}-\mathrm{NMR}$. The functionalization of alginate was confirmed by comparing the IR spectrum of Alg and AlgMA (see Figure 2a) New signals in the Alg-MA spectrum are associated with the vinyl and carbonyl groups. The infrared spectrum of sodium alginate shows signals at $3300 \mathrm{~cm}^{-1}$ (st, OH), $2920 \mathrm{~cm}^{-1}$ (st, C-H), $1633 \mathrm{~cm}^{-1}$ (asymetric st, COO), 1425 $\mathrm{cm}^{-1}$ (symetric st, $\mathrm{COO}$ ). In the AlgMA spectrum all the signals of the alginate are present plus a signal at $1730 \mathrm{~cm}^{-1}$ associated to the stretching of the carbonyl of the ester group. Proton nuclear magnetic resonance spectrum: Figure $2 \mathrm{~b}$ shows the ${ }^{1} H$-NMR spectrum for of Alg and AlgMA. The spectrum of the AlgMA presents the signals at 5.6 and $6.1 \mathrm{ppm}$ attributed to the protons of the vinyl group this molecule, as well as the $1.8 \mathrm{ppm}$ signal corresponds to the hydrogen of the methylene linked to the vinyl group ${ }^{15}$. These results demonstrate unequivocally the functionalization of sodium alginate in a methacrylated macromonomer.

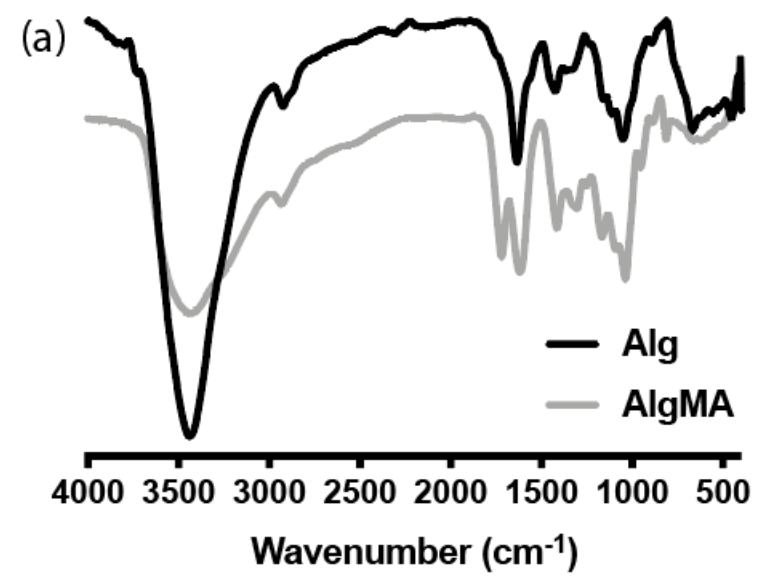

(b)

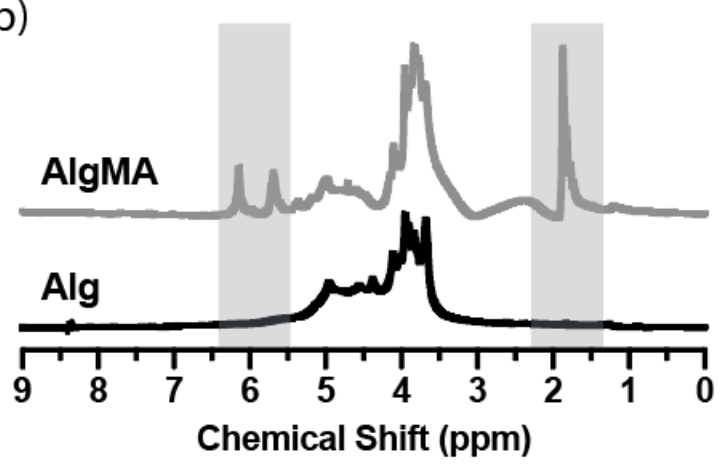

Figure 2. Spectroscopic characterization of sodium alginate (Alg) and methacrylated alginate (AlgMA). (a) FTIR, (b) ${ }^{1} \mathrm{H}-\mathrm{NMR}$. 


\subsection{Rheology characterization.}

To evaluate the viscoelastic properties of the hydrogels, the specimens can be subjected to small deformations, through small amplitude oscillatory shear test (SAOS), that is, the analysis is performed in the linear viscoelastic region where the measured properties are independent of strain or stress applied ${ }^{16}$. The principle of the analysis lies in applying a shear deformation $(\gamma)$ to the sample in a sinusoidal oscillation, $\gamma(t)=\gamma_{0}(\sin \omega t)$, and measuring the generated shear stress $\tau(t)=\tau_{0}(\sin \omega t+\delta)$ which will be out of phase with the deformation, where $\omega$ is the angular frequency and $\delta$ is the phase difference between the two waves. For a purely elastic material, the deformation and stress are in phase, i.e. $\delta=0^{\circ}$, while for a pure liquid the strain and stress are lagged with $\delta=90^{\circ}$. Viscoelastic materials, such as hydrogels, have a phase difference angle between $0<\delta<90^{\circ}$. The critical parameters that are obtained from the rheological analysis informing about the viscoelastic properties of hydrogel are the storage modulus $\left(\mathrm{G}^{\prime}\right)$, the loss modulus $\left(\mathrm{G}^{\prime \prime}\right)$ and the loss tangent $\tan \delta=\mathrm{G}^{\prime \prime} / \mathrm{G}^{\prime}$. $\mathrm{G}^{\prime}$ measures the energy stored during the shearing process and informs about the stiffness of the material, while $G$ "measures the viscous contribution of the material or energy dissipated. If $G^{\prime}>G^{\prime}$ 'the material behaves more elastic, while if $\mathrm{G}^{\prime}<\mathrm{G}^{\prime \prime}$ the sample has more liquid character ${ }^{16}$.

\subsubsection{Optimizing rheological acquisition parameters for hydrogels}

The rheological properties of hydrogels can be very different depending on the composition therefore, it is necessary to optimize acquisition parameters such as geometry size, gap (distance between upper and lower plates), and the solvent trap. For the optimization of these parameters we used a synthetized hydrogel containing $2.0 \mathrm{wt} \%$ of AlgMA, $0.05 \mathrm{wt} \%$ Irgacure as initiator and 10 minutes of polymerization time (see methods).

Diameter of the geometry: The reason for studying the geometries of different dimensions is that increasing the diameter of the geometry the contact surface between the plate and the sample also increase, affecting the stress transfer. Four geometries with sizes of $8,20,25$ and $40 \mathrm{~mm}$ were tested. The hydrogels were synthesized in Teflon molds of the mentioned diameters.
Figure 3 shows the change of the module ( $\mathrm{G}^{\prime}$ and $\mathrm{G}^{\prime \prime}$ ) observing that the geometry of $20 \mathrm{~mm}$ displayed the largest module, followed by the $8 \mathrm{~mm}$ geometry while the $25-$ and $40-\mathrm{mm}$ geometry presented the lowest modules. These results seem to be contradictory since the $40 \mathrm{~mm}$ geometry that has a larger contact surface allows to more efficiently sense the hydrogel deformation resistance. However, there is a variable that cannot be controlled in soft materials, that is the irradiated surface, directly related with the volume of the solution.

Polymerizing in an 8 or $40 \mathrm{~mm}$ diameter mold does not result in the same degree of crosslinking because the volume of macromonomer exposed to UV radiation is not the same and therefore different stiffness may be expected. Focusing only on the geometries of smaller diameter, the geometry of $20 \mathrm{~mm}$ is the one that presents better results, clearly observing the linear viscoelastic region and a less fluctuating of the measurements that can be attributed to the greater contact surface geometry-sample. On the contrary, the geometry of $8 \mathrm{~mm}$ presented a greater variation of the measurements and a constant decrease of $\mathrm{G}^{\prime}$ with the deformation. With these results it was decided to continue with the 20 mm geometry for the next studies.

Gap effect: Figure $3 \mathrm{~b}$ shows the effect of the distance between the plates where the sample is located (the gap). Although the curves are similar in the magnitude of $\mathrm{G}^{\prime}$, there are differences in the extension of the viscoelastic linear region, where a gap of 2.0 the decrease of $\mathrm{G}^{\prime}$ is more accentuated than for $1.5 \mathrm{~mm}$. In other words, when the test is performed at a lower gap, the sample shows greater resistance to deformation. This is attributed to the fact that a smaller gap, the contact between the samples and the geometry is more effective and thus the slippage of the sample with the plate that would lead to an erroneous rheological response is avoided.

Effect of the solvent trap: The function of the solvent trap in this type of tests and especially for materials with high water retention capacity (such as hydrogels) is to avoid the evaporation of the solvent during the test by establishing a thermally stable vapor barrier that makes the sample keep your own solvent. Figure $5 \mathrm{c}$ shows that the effect to the solvent trap is significant, observing that using the trap a module is obtained an order of magnitude greater than without the trap.
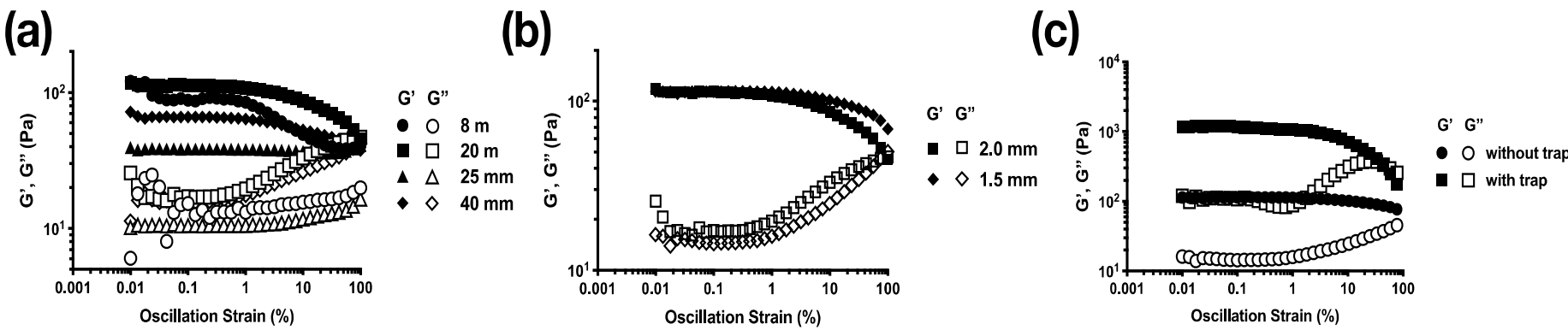

Figure 3. Optimization of rheological acquisition parameters for hydrogels characterization (a) assessment of geometry diameter $\left(\mathrm{gap}=2.0 \mathrm{~mm}, 25^{\circ} \mathrm{C}\right)$. $(\mathrm{b})$ gap effect $\left(\phi=20 \mathrm{~mm}, 25^{\circ} \mathrm{C}\right)$, (c) solvent trap effect $\left(\phi=20 \mathrm{~mm}, 25^{\circ} \mathrm{C}\right)$.

\subsubsection{Assessing synthesis variables.}

Three variables were studied in the photopolymerization of alginate macromonomers: UV exposure time, macromonomer concentration, and photoinitiator concentration.

For the evaluation of the UV exposure time, the hydrogels were prepared with an AlgMA concentration of $2.0 \% \mathrm{~m} / \mathrm{v}$ and $0.05 \% \mathrm{~m} / \mathrm{v}$ of Irgacure. Figure $4 \mathrm{a}$ shows pictures of the hydrogels synthesized with different UV exposure times. The hydrogel with the shortest exposure time $(5 \mathrm{~min})$ has a poor dimensional stability, while the hydrogels polymerized for a longer time reach a more defined and stable geometry. This can be explained by a higher degree of crosslinking by increasing the exposure to UV radiation. In order to assess the changes in degree of crosslinking with polymerization time, the swelling capacity of the hydrogels was studied. It is well known that when a crosslinked polymer is exposed to a affine solvent the polymer chains would expand as a result of the movement of solvent molecules into the polymer due to the difference in osmotic pressure between the solution core and the polymer. However, the expansion of the hydrogel is limited by chain crosslinking that oppose to the complete solubilization. In Figure $4 \mathrm{~b}$ the absorption kinetics for the three gels in Fig. 4a is presented, clearly showing the different water absorption capacities between the hydrogels. The hydrogels polymerized for 5 minutes had a greater swelling than the hydrogel synthesized for $10 \mathrm{~min}$, while gel polymerized for 20 minutes presented the lowest solvent absorption, confirming that by varying the polymerization time a greater crosslinking is achieved.
The viscoelastic properties of the hydrogels were studied by oscillatory strain sweeps tests, evaluating the curves obtained for the storage module $\left(\mathrm{G}^{\prime}\right)$ that informs about the elastic component of the hydrogel and the loss modulus $\left(\mathrm{G}^{\prime \prime}\right)$ associated with the viscous component of the material or energy release by friction. Figure 5 shows the results of the rheological tests for the three synthesis variables studied. Firstly, and in a general way it is observed that for all the hydrogels and at moderate strain $\mathrm{G}^{\prime}>\mathrm{G}^{\prime \prime}$ indicating that the elastic component predominates in accordance with its gel characteristic. Figure 5a shows the effect of the polymerization time on the viscoelastic properties, observing that for both 10 and 20 min of polymerization there is a region where $\mathrm{G}^{\prime}$ does not change with the deformation that corresponds to the linear viscoelastic region and which indicates that the hydrogel behaves elastically without an alteration of its microstructure. Then, the greater deformation occurs a pronounced decrease of $\mathrm{G}$ 'associated with the yield strain of the polymer $\left(\gamma_{\mathrm{s}}\right)$ where the microstructure of the hydrogel begins to be irreversibly altered, while at higher deformation there is a critical strain called flow point where $G^{\prime}=G^{\prime \prime}$, so, on this value the hydrogel will behave like a liquid dissipating a large part of the energy and the microstructure is completely destroyed. Both the $10 \mathrm{mins}$ and the $20 \mathrm{~min}$ polymerized hydrogel have a $\gamma \mathrm{s} \sim 1.0 \%$. A very different composition had the hydrogel synthesized at 5 minutes, where G 'exhibited a constant decrease with the deformation without clearly evidencing the viscoelastic linear region and a storage module significantly lower than the other hydrogels. This indicates a lower stiffness as a result of to a percentage of crosslinking. It is interesting to observe that the flow point of the hydrogel of 5 min occurs at a strain $>100 \%$ while the other hydrogels $\mathrm{G}^{\prime}=\mathrm{G}^{\prime \prime}$ at a strain close to $10 \%$. 
This is explained by the degree of cross-linking achieved, where a greater cross-linking, although it produces a more rigid polymer, also produces a more vitreous material that under a small deformation breaks its microstructure. On the contrary, the less cross-linked hydrogel $(5 \mathrm{~min})$ have greater mobility and consequently they store more energy. In conclusion, the photopolymerization time is a variable that significant influence the properties of the hydrogel. For the following studies, an exposure time of 10 minutes was used.
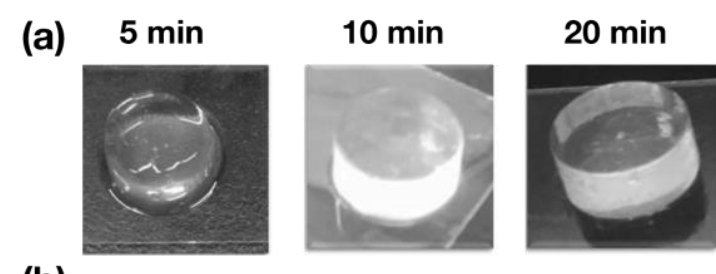

(b)

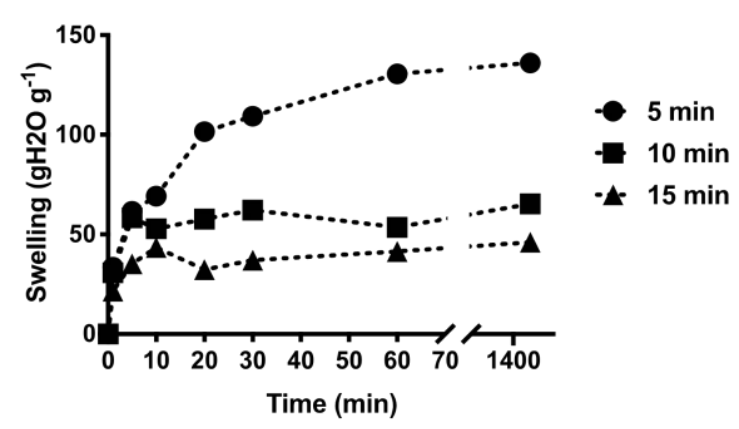

Figure 4. (a) Pictures of hydrogels synthesized at 5, 10 and 15 min of UV exposure. (b) Hydrogels swelling kinetics.

The other variable studied was the concentration of AlgMA. Figure 5b shows the curves of $\mathrm{G}^{\prime}$ and $\mathrm{G}^{\prime \prime}$ for different concentrations of AlgMA. It can be clearly observed high concentration of macromonomer produces a hydrogel with a large modulus. On the other hand, $\gamma_{\mathrm{s}}$ also exhibits differences, while the hydrogel with $1.0 \mathrm{wt} \%$ presents a constant decrease of $\mathrm{G}^{\prime}$ as deformation increased, hydrogels of 2.0 and $3.0 \mathrm{wt} \%$ show a viscoelastic linear region and $\gamma_{\mathrm{s}}=1$ and $10 \%$, respectively. This indicates that the $3.0 \mathrm{wt} \%$ hydrogel resists a greater strain before altering its microstructure. These results can be explained from two approaches: i) the increase in alginate concentration implies an increase in crosslinking sites (methacrylate groups) and ii) greater interaction between alginate chains. Both contribute to an increase in the stiffness of the macromolecular network, the former leading to more sites of chemical crosslinking (covalent) while the latter contributes to a greater crosslinking of a physical type such as entanglements and hydrogen bonds. Finally, the study of the variable concentration of photoinitiator was observed that there is no effect on the rheological properties of the hydrogel. (a)

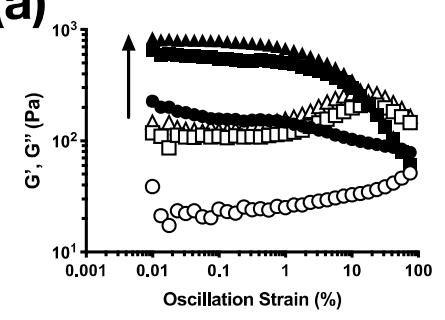

(b)

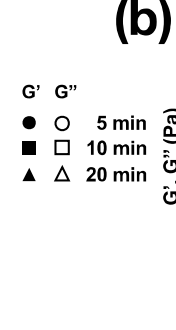

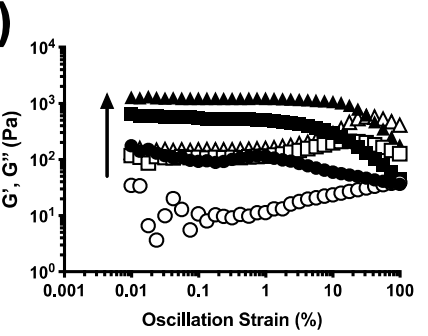

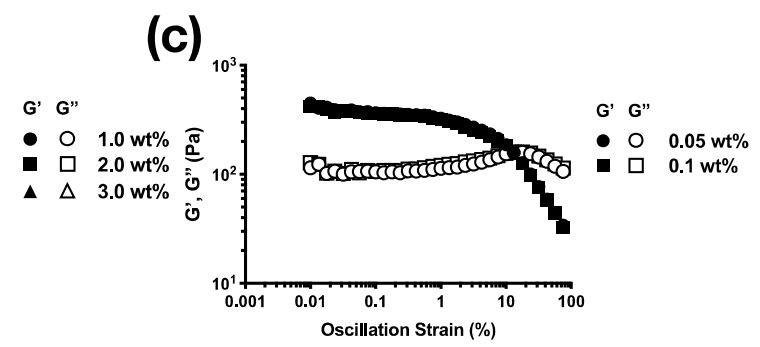

Figure 5. Oscillatory strain sweeps of AlgMA hydrogels. (a) effect of UV exposure time, (b) effect of AlgMA concentration, and (c) effect of Irgacure concentration.

\section{CONCLUSIONS}

In general, when conducting rheological measurements, the efforts are focused on achieving the best contact between the plate and the samples in order to ensure a good sensing. In the particular case of the hydrogels, our data indicate the importance of a preliminary optimization of acquisition parameters (such as diameter of the geometry, gap and solvent trap is particularly) since they significantly change the rheological response. The characterization of synthesis parameters by oscillatory strain sweeps showed that time of exposure to UV radiation and the concentration of the macromonomers have significant effects on the rheological properties of this type of hydrogel. The most important effect was observed for the UV exposure time and the concentration of the macromonomer.

\section{ACKNOWLEDGMENTS}

The authors thank FONDECYT (Grant No 1171082) and FONDEQUIP (EQM 140034). D.A. Palacio thanks the Graduate Direction and Project EDPG173 and University of Concepcion for the doctoral scholarship.

\section{REFERENCES}

1. Ma, S.; Yu, B.; Pei, X.; Zhou, F., Structural hydrogels. Polymer 2016, 98 , 516-535.
2. Guan, X.; Avci-Adali, M.; Alarçin, E.; Cheng, H.; Kashaf, S. S.; Li, Y.; Chawla, A.; Jang, H. L.; Khademhosseini, A., Development of hydrogels for regenerative engineering. Biotechnol. J 2017, 1600394-n/a.

3. Kamata, H.; Li, X.; Chung, U.-i.; Sakai, T., Design of Hydrogels for Biomedical Applications. Adv. Healthc. Mater. 2015, 4 (16), 2360-2374.

4. Caló, E.; Khutoryanskiy, V. V., Biomedical applications of hydrogels: A review of patents and commercial products. Eur. Polym. J. 2015, 65 (Supplement C), 252-267.

5. Geckil, H.; Xu, F.; Zhang, X.; Moon, S.; Demirci, U., Engineering hydrogels as extracellular matrix mimics. Nanomedicine (London, England) 2010, 5 (3), 469-484.

6. Belmar, L.; Toledo, L.; Sánchez, S. A.; Urbano, B. F., Fluorescent nanotubes in PHEMA hydrogels: Visualizing aggregation and distribution by confocal fluorescence microscopy. Materials Today Communications 2018, 16, 285-292.

7. Toledo, L.; Urbano, B. F., Poly(2-hydroxyethyl methacrylate)-based porous hydrogel: Influence of surfactant and $\mathrm{SiO} 2$ nanoparticles on the morphology, swelling and thermal properties. Eur. Polym. J. 2016, 81, 316-326.

8. Passos, M. F.; Dias, D. R. C.; Bastos, G. N. T.; Jardini, A. L.; Benatti, A. C. B.; Dias, C. G. B. T.; Maciel Filho, R., pHEMA hydrogels. J. Therm. Anal. Calorim. 2016, 125 (1), 361-368.

9. Lee, K. Y.; Mooney, D. J., Alginate: Properties and biomedical applications. Prog. Polym. Sci. 2012, 37 (1), 106-126. 
10. Loessner, D.; Meinert, C.; Kaemmerer, E.; Martine, L. C.; Yue, K.; Levett, P. A.; Klein, T. J.; Melchels, F. P. W.; Khademhosseini, A.; Hutmacher, D. W., Functionalization, preparation and use of cell-laden gelatin methacryloyl-based hydrogels as modular tissue culture platforms. Nature Protocols 2016, 11 (4), 727-746.

11. Pourjavadi, A.; Harzandi, A. M.; Hosseinzadeh, H., Modified carrageenan 3. Synthesis of a novel polysaccharide-based superabsorbent hydrogel via graft copolymerization of acrylic acid onto kappa-carrageenan in air. Eur. Polym. J. 2004, 40 (7), 1363-1370.

12. Drury, J. L.; Mooney, D. J., Hydrogels for tissue engineering: scaffold design variables and applications. Biomaterials 2003, 24 (24), 4337-4351.

13. Jeon, O.; Bouhadir, K. H.; Mansour, J. M.; Alsberg, E., Photocrosslinked alginate hydrogels with tunable biodegradation rates and mechanical properties. Biomaterials 2009, 30 (14), 2724-2734.
14. Mignon, A.; Devisscher, D.; Graulus, G.-J.; Stubbe, B.; Martins, J.; Dubruel, P.; De Belie, N.; Van Vlierberghe, S., Combinatory approach of methacrylated alginate and acid monomers for concrete applications. Carbohydr. Polym. 2017, 155, 448-455.

15. Stubbe, B.; Graulus, G. J.; Reekmans, G.; Courtin, T.; Martins, J. C.; Van Vlierberghe, S.; Dubruel, P.; Adriaensens, P., A straightforward method for quantification of vinyl functionalized water soluble alginates via 13C-NMR spectroscopy. Int. J. Biol. Macromol. 2019, 134, 722-729.

16. Mezger, T. G., The Rheology Handbook: For Users of Rotational and Oscillatory Rheometers. Vincentz Network: 2006. 